\title{
Synergistic anti-methicillin-resistant Staphylococcus aureus (MRSA) activity and absolute stereochemistry of 7,8-dideoxygriseorhodin C
}

\author{
Bailey W. Miller $\mathbb{1}^{1} \cdot$ Joshua P. Torres $^{2} \cdot$ Jortan O. Tun $^{2} \cdot$ Malem S. Flores $^{2} \cdot$ Imelda Forteza $^{2} \cdot$ Gary Rosenberg $^{3} \cdot$ \\ Margo G. Haygood ${ }^{1}$ • Eric W. Schmidt ${ }^{1}$ - Gisela P. Concepcion ${ }^{2}$
}

Received: 10 October 2019 / Revised: 16 December 2019 / Accepted: 20 December 2019 / Published online: 28 January 2020

(c) The Author(s) 2020. This article is published with open access

\begin{abstract}
The emergence of antibiotic resistance necessitates not only the identification of new compounds with antimicrobial properties, but also new strategies and combination therapies to circumvent this growing problem. Here, we report synergistic activity against methicillin-resistant Staphylococcus aureus (MRSA) of the $\beta$-lactam antibiotic oxacillin combined with 7,8-dideoxygriseorhodin $\mathrm{C}$ in vitro. Ongoing efforts to identify antibiotics from marine mollusk-associated bacteria resulted in the isolation of 7,8-dideoxygriseorhodin C from a Streptomyces sp. strain cultivated from a marine gastropod tissue homogenate. Despite the long history of 7,8-dideoxygriseorhodin $\mathrm{C}$ in the literature, the absolute configuration has never been previously reported. A comparison of measured and calculated ECD spectra resolved the configuration of the spiroketal carbon C6, and 2D ROESY NMR spectroscopy established the absolute configuration as 6s,6aS. The compound is selective against Gram-positive bacteria including MRSA and Enterococcus faecium with an MIC range of $0.125-0.5 \mu \mathrm{g} \mathrm{ml}^{-1}$. Moreover, the compound synergizes with oxacillin against MRSA as observed in the antimicrobial microdilution and time-kill assays. Simultaneous treatment of the compound with oxacillin resulted in an approximately tenfold decrease in MIC with a combination index of $<0.5$, indicating synergistic anti-MRSA activity.
\end{abstract}

\section{Introduction}

Marine gastropod mollusks that are defended by thick shells harbor diverse culturable bacteria, many of which produce biologically active compounds [1-4]. Our continuing efforts to screen extracts from mollusk-associated bacteria against methicillin-resistant Staphylococcus aureus (MRSA) led us to

These authors contributed equally: Bailey W. Miller, Joshua P. Torres, Jortan O. Tun

Supplementary information The online version of this article (https:// doi.org/10.1038/s41429-019-0275-8) contains supplementary material, which is available to authorized users.

Gisela P. Concepcion

gpconcepcion@up.edu.ph

1 Department of Medicinal Chemistry, University of Utah, Salt Lake City, UT 84112, USA

2 The Marine Science Institute, University of the Philippines Diliman, Quezon City 1101, Philippines

3 Academy of Natural Sciences Philadelphia, Drexel University, 1900 Benjamin Franklin, Parkway, PA 19103, USA the isolation of 7,8-dideoxygriseorhodin C (1) from Streptomyces sp. strain 1425S.R.1a.1. The bacterium was isolated from the body tissue homogenate of Truncatella guerinii (Fig. 1), a small gastropod that lives under plant debris near splash zones. Griseorhodins are aromatic polyketides known to be biologically active against Gram-positive bacteria [5-8]. In addition, some members of this structural family are known to be inhibitors of HIV reverse transcriptase and human telomerases [9]. The most recently isolated griseorhodins D, E, and $\mathrm{F}$ are intermediates and end products of post-PKS tailoring modification during biosynthesis [1].

Previously, $S$. aureus infections were primarily treated with $\beta$-lactam antibiotics, including oxacillin, a secondgeneration penicillin. Soon after the emergence of MRSA, these early generation $\beta$-lactam antibiotics were eliminated from the treatment options. To date, MRSA has become one of the most prevalent multi-drug resistant pathogens, and is responsible for most nosocomial and community-acquired infections worldwide [10, 11]. Currently, antibiotic treatments for MRSA infections are limited to glycopeptides (vancomycin and telavancin), oxazolidinones (linezolid and tedizolid), lipopeptides (daptomycin), and advancedgeneration $\beta$-lactams, such as cephalosporins (ceftaroline). 


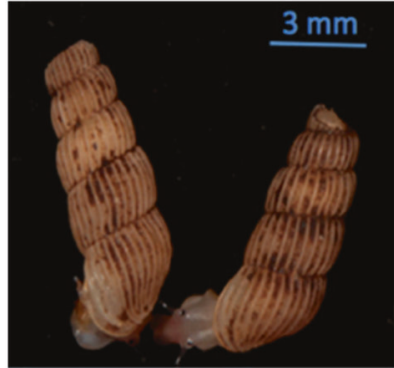

(a)

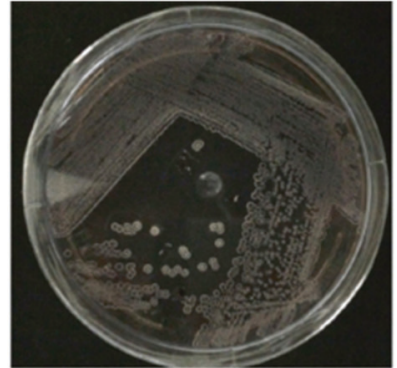

(b)
Fig. 1 Animal and microbial sources of 1. a Truncatella guerinii collected in Cebu, Philippines. b Streptomyces sp. (1425S.R.1a.1) grown on $\mathrm{R} 2 \mathrm{~A}$ agar with $2 \% \mathrm{NaCl}$ isolated from the body tissue homogenate

Development of resistance to any antibiotic is inevitable. Apart from discovering new classes of antibiotics, combination drug therapy is another strategy to prevent or minimize the development of drug resistance [12]. Thus, we have begun to test compounds isolated from marine mollusk-associated bacteria that show anti-MRSA activity in combination with known antibiotics to identify potential synergistic interactions. When MRSA (ATCC ${ }^{\circledR} 43300^{\mathrm{TM}}$ ) was treated simultaneously with 7,8-dideoxygriseorhodin C and oxacillin, a more potent antimicrobial activity was observed compared with the single-drug treatments. This indicates possible synergistic activity, which may translate to a more rapid clearance of infection, shorter course of antibiotic therapy, and therefore, a reduction of dose-related toxicity [13].

Despite the long history of $\mathbf{1}$ in the literature, the absolute stereochemistry has never been reported. In addition, NMR chemical shifts were originally assigned without modern high-field NMR technology and advanced 2D experiments, and comparison with reported shift assignments for members of the structural family revealed inconsistently assigned signals. All carbon chemical shift signals were assigned, and the stereochemistry was determined to be $6 S, 6 \mathrm{a} S$ by using a combination of spectroscopic and computational methods.

\section{Materials and methods}

\section{General experimental procedures}

UV-Vis spectra were obtained by using a Shimadzu Prominence HPLC System (Shimadzu, Kyoto, Japan) coupled with a PDA detector. High-resolution electrospray ionization mass spectra were gathered using a QSTAR $^{\circledR}$ XL Hybrid LC/MS/MS System (Applied Biosystems, Foster City, CA, USA) equipped with turbo ion spray source, delivering the sample at a rate of $40 \mu \mathrm{min}^{-1}$. NMR data were collected using a Varian 500-MHz NMR spectrometer with $5 \mathrm{~mm}$ Varian Oneprobe $\left({ }^{1} \mathrm{H} 500 \mathrm{MHz},{ }^{13} \mathrm{C} 125 \mathrm{MHz}\right)$. Residual signals from solvents were used for referencing. Semipreparative HPLC was performed using a Phenomenex C18 column (Luna $250 \times 10 \mathrm{~mm}, 5 \mu \mathrm{m}, 4.0 \mathrm{ml} \mathrm{min}^{-1}$ ). ECD spectra were obtained on an Aviv Biomedical Model 410 CD Spectrometer.

\section{Collection of animal material}

Truncatella guerinii (specimen code PMS-1419Y) (Fig. 1) was collected in Tambuli East, Lapu-Lapu City, Cebu, Philippines in September 2009 and January 2011. Appropriate permits from the local government and the Bureau of Fish and Aquatic Resources (BFAR) were secured prior to sample collection. Two voucher specimens stored in $95 \%$ ethanol were prepared and deposited at the Marine Science Institute Museum and the Malacology Department of the Academy of Natural Sciences of Drexel University. Samples were immediately processed under sterile conditions in a temporary laboratory set-up at the collection site. A pool of five specimens was washed three times with sterile water before cracking the shells using a shell vise. Samples were dissected by Dr Alexander Fedosov of A.N. Severtzov Institute of Ecology and Evolution of the Russian Academy of Science. Individual tissues were extracted including the prostate gland, digestive tract, and the remainder of the body. Samples were placed in $1.5-\mathrm{ml}$ microcentrifuge tubes and kept in an ice bath until microbial isolation.

\section{Isolation and identification of bacteria}

After dissection, each tissue sample was homogenized in sterile sea water $(1 \mathrm{ml})$ using mortar and pestle. The resulting homogenate was serially diluted (tenfold) and plated on R2A (Reasoner's $2 \mathrm{~A})$ agar $(0.2 \%$ yeast extract, $1 \%$ malt extract, $0.2 \%$ glucose) supplemented with $2 \%$ $\mathrm{NaCl}, 10 \mu \mathrm{g} \mathrm{ml}^{-1}$ nalidixic acid, $10 \mu \mathrm{g} \mathrm{ml}^{-1}$ cycloheximide, and $25 \mu \mathrm{g} \mathrm{ml}^{-1}$ nystatin. Plating for each dilution was done in duplicate inside a portable hood that was sterilized using UV and $70 \%$ ethanol. Primary or "mother" plates were incubated for 4 weeks at $30^{\circ} \mathrm{C}$. Bacteria were isolated, purified, and preserved in $20 \%$ glycerol at $-80{ }^{\circ} \mathrm{C}$. Bacteria with the code 1425S.R.1a.1 were isolated from the body of T. guerinii (specimen code PMS-1419Y). Genomic DNA of 1425S.R.1a.1. was extracted from a bacterial culture $(7 \mathrm{ml})$ using the GenCatch ${ }^{\mathrm{TM}}$ Blood \& Tissue Genomic Mini-Prep Kit (Epoch Life Science) following the manufacturer's protocol. The 16S rRNA gene was amplified by polymerase chain reaction (PCR) using 20-50 ng template DNA, $6 \mu$ of 2X PCR Master Mix (Promega), and $0.5 \mu \mathrm{M}$ of primers: 27F (5'-AGAGTTTGATCCTGGCTCAG-3') and $1492 \mathrm{R}$ (5'-TACGGYTACCTTGTTACGACTT-3'), for a total reaction volume of $20 \mu \mathrm{l}$. Thermal cycling consisted of an 
initial denaturation step at $95^{\circ} \mathrm{C}$ for $3 \mathrm{~min}$, followed by 30 cycles of $95^{\circ} \mathrm{C}$ for $30 \mathrm{~s}, 48^{\circ} \mathrm{C}$ for $20 \mathrm{~s}, 72^{\circ} \mathrm{C}$ for $1 \mathrm{~min}$, and then a final extension step at $72{ }^{\circ} \mathrm{C}$ for $7 \mathrm{~min}$. The amplicon was purified using the GenCatch ${ }^{\mathrm{TM}}$ Advanced PCR extraction kit (Epoch Life Science) and subsequently evaluated for correct size ( $1400 \mathrm{bp})$ and homogeneity using $1 \%$ agarose gel electrophoresis before sending to Macrogen, Inc. (Seoul, South Korea) for gene sequencing. Two additional internal primers 518F (5'-CCAGCAGCCGCGGTAATACG- $3^{\prime}$ ) and 800R (5'-TACCAGGGTATCTAA TCC-3') were used in sequencing. The four fragments were assembled and annotated using Geneious 8.1 software (Biomatters, New Zealand, available at http://www. geneious.com). Subsequently the contig was aligned against Ribosomal Database Project and GenBank nucleotide sequence databases using BLAST to identify similar bacterial 16S rRNA sequences. BLAST revealed that 1425S.R.1a.1 was $99 \%$ similar to Streptomyces sp. The $16 \mathrm{~S}$ rDNA sequence was deposited in GenBank with accession code KY906645.

\section{Fermentation, extraction, and isolation}

Streptomyces sp. (1425S.R.1a.1) was inoculated from a seed culture $(100 \mathrm{ml})$ into eighteen $2.8 \mathrm{~L}-$ Fernbach flasks, each containing 11 of R2A broth $(0.2 \%$ yeast extract, $1 \%$ malt extract, $0.2 \%$ glucose, and supplemented with $2 \%$ $\mathrm{NaCl}$ ), and cultured for 6 days at $30^{\circ} \mathrm{C}$ with shaking at 150 $\mathrm{rpm}$. The fermentation broth was centrifuged at $4000 \mathrm{rpm}$ at $20^{\circ} \mathrm{C}$ to separate the supernatant from the mycelia. The supernatant was extracted with HP20 diaion resin for $3 \mathrm{~h}$. The diaion resin was recovered through filtration and washed with $\mathrm{dH}_{2} \mathrm{O}$ to remove salt before eluting the resin with $25,50,75$, and $100 \%$ methanol $(\mathrm{v} / \mathrm{v})$. The methanol fraction was concentrated under reduced pressure and extracted with ethyl acetate. Finally, the ethyl acetate fraction was concentrated under reduced pressure to give the crude extract $(620 \mathrm{mg})$. The crude extract was separated into six fractions on a $\mathrm{C} 18$ open column using a step gradient elution of acetonitrile and $\mathrm{H}_{2} \mathrm{O}$ with $0.1 \%$ TFA $(20,30,40$, $50,60,70,80,90$, and 100\%). Fraction 8 (90\% acetonitrile $(\mathrm{ACN})$ ), which was active against MRSA, was subjected to silica gel column chromatography using $(9.5: 0.5)$ dichloromethane/methanol $/ 0.1 \%$ acetic acid to yield bioactive fractions 9-3, 9-4, and 9-5 which were pooled $(8.0 \mathrm{mg})$ and further purified by semipreparative HPLC (70\% ACN: $\mathrm{H}_{2} \mathrm{O}$ $+0.05 \% \mathrm{TFA})$ to give 7,8-dideoxygriseorhodin $\mathrm{C}(1.7 \mathrm{mg})$.

7,8-dideoxygriseorhodin $\mathrm{C}$ (1) was obtained as a red powder, UV $(\mathrm{MeOH}+0.01 \% \mathrm{TFA}): \lambda_{\max } 230,256,312$, 352, 480, 505, $548 \mathrm{~nm} ;{ }^{1} \mathrm{H}$ and ${ }^{13} \mathrm{C}$ NMR (Table S1); HRESIMS $m / z=495.0889 \quad[\mathrm{M}+\mathrm{H}]^{+}$calculated for $\mathrm{C}_{25} \mathrm{H}_{19} \mathrm{O}_{11}, 495.0927$.

\section{Computational methods for ECD prediction}

DFT calculations were performed at $298 \mathrm{~K}$ in methanol solution using the polarizable continuum model (PCM) in its integral equation formalism version (IEFPCM) incorporated into Gaussian 16 software. Models of all four diastereomers $(6 S, 6 \mathrm{a} S ; 6 R, 6 \mathrm{a} S ; 6 R, 6 \mathrm{a} R ; 6 S, 6 \mathrm{a} R)$ were generated in Chem3D 15.0 (PerkinElmer). Each was energy minimized in Spartan (Wavefunction Inc.), and a conformer search was carried out at the molecular mechanics level of theory. Boltzmann distribution tables were generated for the top ten conformers of each diastereomer. The conformers that represented a combined $>99 \%$ of the population were geometry optimized at the b3lyp/6-31 g(d,p) level. TD-DFT calculations were performed at the b3lyp/6-31g $\left(\mathrm{d}^{\prime}, \mathrm{p}^{\prime}\right)$ level with the number of states set to $N=40$. Transitions were loaded into SpecDis 1.71 [14] for the generation of ECD spectra, and the spectrum from each conformer was weighted according to the Boltzmann distribution to produce a final averaged spectrum for each diastereomer.

\section{Computational methods for ${ }^{13} \mathrm{C}$ NMR predictions}

The top configurations of $6 S, 6 \mathrm{a} S$ and $6 R, 6 \mathrm{a} S$ were loaded into Gaussian 16. QM-NMR predictions were performed using the GIAO Method at the b3lyp/6-31g $\left(\mathrm{d}^{\prime}, \mathrm{p}^{\prime}\right)$ level. Trimethylsilane (TMS) was modeled, optimized, and run with the same methods and at the same level of theory to be used as a reference.

\section{Determination of minimum inhibitory concentration (MIC)}

The protocol was based on the Clinical Laboratory Standards Institute M7-A7 [15], with some modifications. Colonies of MRSA (ATCC ${ }^{\circledR} 43300^{\mathrm{TM}}$ ) were inoculated in $10 \mathrm{ml}$ of cation-adjusted Meuller-Hinton broth (CAMHB) with $2 \% \mathrm{NaCl}$ and incubated for $6 \mathrm{~h}, 37^{\circ} \mathrm{C}$, with shaking at $150 \mathrm{rpm}$. The turbidity of the bacterial suspension was adjusted with sterile broth to absorbance values of $0.08-0.1$ at $625 \mathrm{~nm}$ to achieve a suspension containing $\sim 1 \times 10^{8} \mathrm{CFU}$ $\mathrm{ml}^{-1}$. The adjusted inoculum was further diluted 100-fold and used for the assay. The inoculum was added into a 96well flat-bottom microtiter plate containing $98 \mu \mathrm{l}$ of CAMHB with $2 \% \mathrm{NaCl}$ and $2 \mu \mathrm{l}$ of each drug concentration. The final density of the bacteria per well was $\sim 5 \times 10^{5}$ $\mathrm{CFU} \mathrm{ml}{ }^{-1}$. Afterward, the plate was sealed and incubated with shaking for $18-24 \mathrm{~h}$ at $37{ }^{\circ} \mathrm{C}$. Finally, $20 \mu \mathrm{l}$ of $0.02 \%$ resazurin was added into each well and the fluorescence signals were measured at $530 \mathrm{~nm}$ excitation and $590 \mathrm{~nm}$ emission using a microplate reader (Biotek Synergy ${ }^{\mathrm{TM}}$ HT, VT, USA). MRSA treated with oxacillin and vehicle 
solvent was used as positive and growth controls, respectively. The concentration of DMSO per well did not exceed $1 \%$. The results of the assays were expressed as \% inhibition relative to the negative control using the following equation:

$\%$ Inhibition $=\left(1-\left(\frac{\text { Absorbance treated }- \text { Absorbance media }}{\text { Absorbance vehicle }- \text { Absorbance media }}\right)\right) \times 100$

The data points were plotted in GraphPad Prism version 5 and inputted in CompuSyn software [16] for the determination of MIC. The MIC is defined as the lowest concentration of the antimicrobial agent that inhibits $97-100 \%$ of growth.

\section{Evaluation of synergistic activity}

The synergistic activity of 7,8-dideoxygriseorhodin $\mathrm{C}$ with oxacillin was determined by performing the antimicrobial microdilution broth assay as described above. MRSA was treated with serial twofold concentrations of the compounds concurrently at constant $1 \mathrm{X}$ MIC ratio. The MIC of the individual drugs was also determined simultaneously as single-drug controls. To determine synergism or antagonism, the combination index (CI) at $\mathrm{MIC}_{97}$ was analyzed using CompuSyn [16] based on the equation below [17, 18]. $\mathrm{CI} \leq 0.5,>0.5-4,>4$ mean synergism, additive, antagonism, respectively [19].

$\mathrm{CI}=\frac{\mathrm{MIC}_{\mathrm{a}} \text { combination }}{\mathrm{MIC}_{\mathrm{a}} \text { individual }}+\frac{\mathrm{MIC}_{\mathrm{b}} \text { combination }}{\mathrm{MIC}_{\mathrm{b}} \text { individual }}$

\section{Time-kill assay}

Time-kill assays were done in 96-well microplates. MRSA $\left(5 \times 10^{4} \mathrm{CFU} /\right.$ well $)$ was treated singly and in combination with $1\left(0.0625\right.$ and $\left.0.125 \mu \mathrm{g} \mathrm{ml}^{-1}\right)$ and oxacillin $(4$ and $8 \mu \mathrm{g}$ $\mathrm{ml}^{-1}$ ), and then $10 \mu \mathrm{l}$ were aliquoted in each well at $2,4,8$, 12 , and $24 \mathrm{~h}$ post treatment, and serially diluted by tenfold. Ten microliters of each dilution were spotted on antibioticfree CAMHBII agar plates. Colonies from the last dilution with 10-100 colonies were counted after 20-24 h of incubation, and the $\mathrm{CFU} \mathrm{ml}{ }^{-1}$ was calculated. Each experiment was conducted in triplicate. Synergy was defined as $\geq 2$ $\log 10$ decrease in colony counts at $24 \mathrm{~h}$ with the combination, compared with the most active single-drug alone.

\section{MTT cell viability assay}

Quantification of cell viability was based on the metabolism of MTT (3-(4,5-dimethyl-2-thiazolyl)-2,5-diphenyl-2Htetrazolium bromide) by the live cells [20]. MDCK (Madin-Darby canine kidney cells) ATCC ${ }^{\circledR}$ CCL-34 ${ }^{\mathrm{TM}}$ and AA8 (Chinese hamster ovarian cells) ATCC ${ }^{\circledR}$ CRL-1859 ${ }^{\text {TM }}$ were used as in vitro models for toxicity testing. Briefly, cells were seeded into a flat-bottom 96-well plate at a density of $2 \times 10^{4}$ cells/well and incubated at $37^{\circ} \mathrm{C}$ for $24 \mathrm{~h}$. Subsequently, cells were treated with 1, doxorubicin (positive control) or vehicle control (1\% DMSO) and exposed for $72 \mathrm{~h}$. Afterward, the contents of the wells were discarded by sharp flicking the plate. Then, $15 \mu$ of filtered MTT ( $5 \mathrm{mg} \mathrm{ml}^{-1}$ in 1X PBS) was added to each well and incubated at $37^{\circ} \mathrm{C}, 5 \% \mathrm{CO}_{2}$ for another $3 \mathrm{~h}$. Finally, the formazan crystals formed from the reaction were solubilized with $100 \mu \mathrm{l}$ of DMSO per well. After $5 \mathrm{~min}$ of shaking, the absorbance was read in a microplate reader (Biotek $^{\circledR}$, Synergy-HT, VT, USA) at $570 \mathrm{~nm}$. The results of the assays were expressed as percent cell viability relative to the negative controls using the following equation:

$\%$ Cell viability $=\left(\frac{\text { Absorbance treated }- \text { Absorbance media }}{\text { Absorbance vehicle }- \text { Absorbance media }}\right) \times 100$

\section{Results and discussion}

\section{Isolation, structure verification, and chemical shift reassignment}

A Streptomyces sp. bacterium was isolated from the body tissue homogenate of a marine mollusk, T. guerinii obtained from Cebu, Philippines. The bacterium was grown in 181 of R2A media supplemented with $2 \% \mathrm{NaCl}$, the cells were removed by centrifugation, and the culture medium was extracted with hydrophobic resin. The resin was washed, and then eluted with $\mathrm{MeOH}$ to produce a dark red crude extract that showed antibacterial activity against methicillinresistant S. aureus (MRSA, ATCC ${ }^{\circledR} 43300^{\mathrm{TM}}$ ). Assayguided purification of active constituents provided a series of red pigments, most of which appeared to be known analogs of the griseorhodin family based on spectroscopic data.

The most hydrophobic compound, based on retention in C18 reversed-phase chromatography, was isolated as a red solid. High-resolution mass spectrometry showed a protonated molecule at $m / z=495.0889(\mathrm{M}+\mathrm{H})^{+}$, suggesting a molecular formula of $\mathrm{C}_{25} \mathrm{H}_{18} \mathrm{O}_{11}$ with 17 degrees of unsaturation (Fig. S1), consistent with the formula of 7,8dideoxygrisheorhodin $\mathrm{C}(\mathbf{1})$. Furthermore, the $1 \mathrm{D}^{13} \mathrm{C}$ NMR experiment and UV-Vis absorption spectra matched the reported values for 7,8-dideoxygrisheorhodin C [21]. The ${ }^{1} \mathrm{H}$ NMR spectrum, however, revealed slight changes in the chemical shifts of the two pairs of methylene protons on carbons 7 and 8 (Table S1). 2D NMR experiments, including gCOSY, gHSQCAD, and gHMBCAD, were used to verify the planar structure to be identical to previous reports (Fig. 2). 


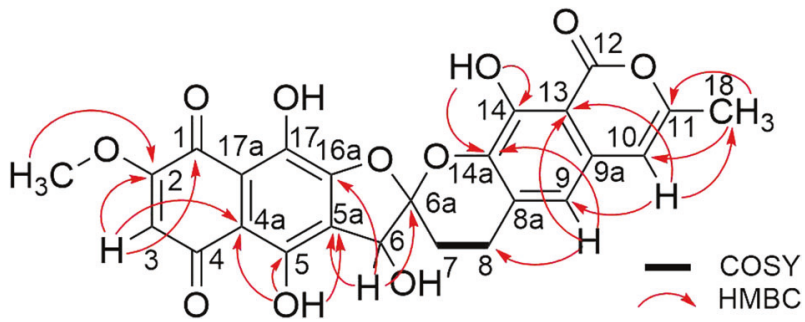

Fig. 2 Key NMR correlations for the structural assignment of $\mathbf{1}$

Table 1 Carbon chemical shift reassignment in 7,8 dideoxygriseorhodin $\mathrm{C}$

\begin{tabular}{|c|c|c|c|}
\hline Position & $\begin{array}{l}(1) \\
\delta_{\mathrm{C}}\end{array}$ & $\begin{array}{l}\text { Original [21] } \\
\delta_{\mathrm{C}}\end{array}$ & $\begin{array}{l}\text { Calculated } \\
\delta_{\mathrm{C}}\end{array}$ \\
\hline 1 & 180.5 & 179 & 184.2 \\
\hline 2 & 160.7 & 160.1 & 161 \\
\hline $2-\mathrm{OCH}_{3}$ & 57.5 & 56.94 & 57.6 \\
\hline 3 & 110.6 & 110.1 & 111.4 \\
\hline 4 & 186.1 & 184.5 & 185.8 \\
\hline $4 a$ & 106.9 & 106.3 & 109.6 \\
\hline 5 & 157.5 & 158 & 154.8 \\
\hline $5 a$ & 124.5 & 130.2 & 126.8 \\
\hline 6 & 74.5 & 74.1 & 80.5 \\
\hline $6 \mathbf{a}$ & 113.6 & 152.1 & 118.5 \\
\hline 7 & 23.6 & 21.3 & 28.3 \\
\hline 8 & 21.7 & 23.2 & 27.5 \\
\hline $8 a$ & 132.9 & 124.2 & 134.3 \\
\hline 9 & 115.4 & 114.8 & 114.5 \\
\hline $9 a$ & 130.7 & 124.2 & 131.4 \\
\hline 10 & 104.2 & 103.7 & 107.1 \\
\hline 11 & 152.7 & 148.7 & 155 \\
\hline 12 & 166.1 & 165.6 & 166.6 \\
\hline 13 & 104.7 & 113.3 & 106.5 \\
\hline 14 & 149.1 & 147.9 & 150.2 \\
\hline $14 a$ & 138.2 & 130.2 & 137.8 \\
\hline $16 \mathbf{a}$ & 147.1 & 137.8 & 146.8 \\
\hline 17 & 154.1 & 154.1 & 153.9 \\
\hline $17 \mathrm{a}$ & 114.5 & 113.9 & 117.3 \\
\hline 18 & 19.1 & 18.5 & 22.6 \\
\hline
\end{tabular}

Shifts that were reassigned based on 2D NMR experiments are shown in bold, while shifts that did not have 2D NMR correlations, reassigned purely by comparison with calculated values are in italics

As previously stated, the ${ }^{13} \mathrm{C}$ NMR spectrum was identical to reports in the literature. However, during the structure verification it became clear that there were significant misassignments of chemical shifts, particularly within the proton-deficient polycyclic regions. This is likely due to the original reports relying solely on $1 \mathrm{D}{ }^{1} \mathrm{H}$ and ${ }^{13} \mathrm{C}$ NMR data, without the aid of modern 2D experiments.
The original report lists the ${ }^{13} \mathrm{C}$ and ${ }^{1} \mathrm{H}$ shifts at $\mathrm{C} 7$ as 21.30 and $1.99 / 1.92 \mathrm{ppm}$, while $\mathrm{C} 8$ was $\delta 23.24$ and $2.51 /$ $2.43 \mathrm{ppm}$. However, it is clear from the HSQC data that the more upfield proton set $(\delta 2.16 / 2.40)$ should be associated with the carbon signal at $\delta 23.6 \mathrm{ppm}$, while the methylene protons at $\delta 3.05$ are on the carbon at $\delta 21.7 \mathrm{ppm}$ (Fig. S4). An HMBC correlation from $\mathrm{H} 9(\delta 6.86)$ to the carbon at $\delta$ 21.7 supports this being $\mathrm{C} 8$, while $\mathrm{C} 7$ is at $\delta$ 23.6. Furthermore, the different chemical shifts for the protons on C7 support this being adjacent to the C6a stereocenter, while the protons on $\mathrm{C} 8$ are seen as a single peak with an integration of 2 . These results mirror those of the structurally related compound hyaluromycin, in which the proton chemical shifts at $\mathrm{C} 7$ are also more upfield in comparison with those at $\mathrm{C} 8$ [22]. The chemical shifts of the nonprotonated carbons $\mathrm{C} 1, \mathrm{C} 2, \mathrm{C} 4, \mathrm{C} 4 \mathrm{a}, \mathrm{C} 5, \mathrm{C} 12$, and $\mathrm{C} 17$ were confirmed, while carbons C5a, C6a, C11, C13, C14, $\mathrm{C} 14 \mathrm{a}$, and $\mathrm{C} 16 \mathrm{a}$ were all reassigned, based on $\mathrm{HMBC}$ correlations (Fig. 2).

In addition, six carbon nuclei (C4, C8a, C9a, C12, C17, and $\mathrm{C} 17 \mathrm{a})$ remained with no $2 \mathrm{D}$ NMR correlations to validate chemical shift assignment. Thus, QM-DFT calculations were run in Gaussian 16 to predict the ${ }^{13} \mathrm{C}$ NMR chemical shifts for all nuclei in the compound. The results increased confidence for the placements of the 20 carbons assigned based on 2D NMR data. The remaining six carbons were all able to be assigned based on their relative shift values and confirmed with comparison with the calculated results (Table 1).

Taken together, these data verify that the isolated compound is the same planar structure as the originally reported 7,8-dideoxygriseorhodin C. Furthermore, a combination of experimental and computational methods corroborated the reassignment of chemical shift data to the planar structure of the molecule. However, there remains a discrepancy in the proton shifts for the two methylene groups between the original report of 7,8-dideoxygriseorhodin $\mathrm{C}$ and our report here. Because the original papers did not report any stereochemical analysis, it is possible that the discrepancy arises from a difference in configuration between the original isolation and the material reported herein.

\section{Absolute configuration of 7,8-dideoxygriseorhodin C}

Two stereocenters are present in $\mathbf{1}$ at carbons 6 and 6 a. Interestingly, previous reporting on the absolute configurations of griseorhodins $\mathrm{A}$ and $\mathrm{C}$ showed the configuration of carbon 6a to be $S$ in both compounds, while carbon 6 is identified as $S$ in griseorhodin A and $R$ in griseorhodin C $[23,24]$. Despite being known for many years, no reports have been made on the absolute or relative configuration of 7,8-dideoxygriseorhodin C. 


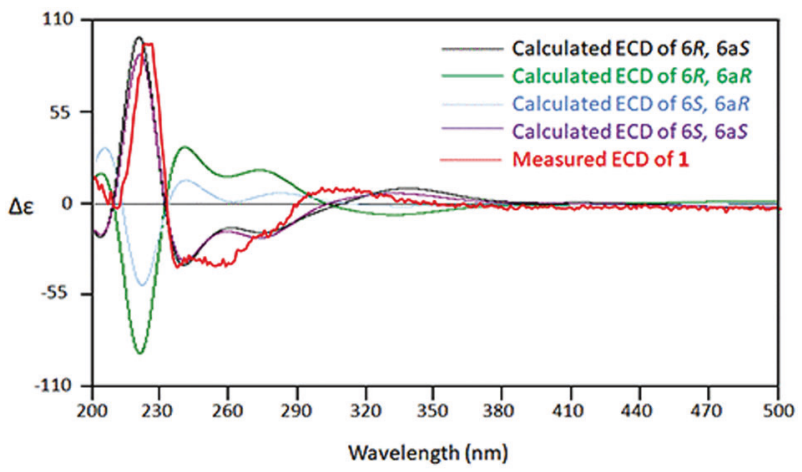

Fig. 3 Experimentally derived (red) vs. calculated ECD spectra of each possible diastereomer of 7,8-dideoxygriseorhodin C. 6R,6aS (black) and $6 S, 6 \mathrm{a} S$ (purple) both match the experimental data, but are not distinguishable from one another

Stereochemical assignment was done through a combination of calculated vs. experimental ECD spectra and through-space NOE correlations. The four possible diastereomers $(6 R, 6 \mathrm{a} R ; 6 R, 6 \mathrm{a} S ; 6 S, 6 \mathrm{a} R ; 6 S, 6 \mathrm{a} S)$ were modeled and imported to Spartan to perform a conformer search. The free energy of each conformer was used to create a Boltzmann distribution, and those that represented a combined $>99 \%$ of the population of each diastereomer were imported to Gaussian 16. These were geometry optimized, and TDDFT calculations were performed to calculate transitions, which were imported into SpecDis 1.71 for the generation of calculated ECD spectra. Spectra were weighted according to the Boltzmann distribution and combined to create a single, averaged spectrum for each diastereomer, then compared with the experimental result (Fig. 3).

The stereocenter at carbon 6a has a dramatic effect on the calculated ECD spectrum, clearly supporting the assignment of carbon $6 \mathrm{a}$ as the $S$ configuration, which is consistent with the previous reports within this structural class $[23,24]$. The calculated spectra for $6 S, 6 \mathrm{a} S$ and $6 R, 6 \mathrm{a} S$, however, are indistinguishable by this method, leaving the configuration of $\mathrm{C} 6$ ambiguous.

Based on the 3D modeling, the configuration of C6 will have a significant effect on the spatial relationships between $\mathrm{H} 6,6-\mathrm{OH}$, and the diastereotopic protons $\mathrm{H} 7 \mathrm{a}$ and $\mathrm{H} 7 \mathrm{~b}$. In the model for $6 R, 6 \mathrm{aS}, \mathrm{H} 6$ is oriented towards $\mathrm{H} 7 \mathrm{a}$ and $\mathrm{H} 7 \mathrm{~b}$ within $2.8 \AA$ of both, while 6-OH is pointed away. Alternatively, in $6 S, 6 \mathrm{a} S$, the hydroxyl group is in the position close to the $\mathrm{C} 7$ protons, while $\mathrm{H} 6$ is 3.17 and $3.68 \AA$ away from $\mathrm{H} 7 \mathrm{a}$ and $\mathrm{H} 7 \mathrm{~b}$, respectively (Fig. 4b). The ROESY spectrum shows a clear cross-peak between the C6 hydroxyl proton $\left(\delta_{\mathrm{H}} 6.42\right)$ with both $\mathrm{H} 7 \mathrm{a}$ and $\mathrm{H} 7 \mathrm{~b}\left(\delta_{\mathrm{H}} 2.16\right.$ and 2.40$)$ (Fig. 4a). These cross-peaks are in the positive phase, while 6-OH cross-peaks with the phenolic protons are in the negative phase, indicating real NOE correlations. H6 $\left(\delta_{\mathrm{H}}\right.$ 5.12), however, has a weaker cross-peak with $\mathrm{H} 7 \mathrm{a}$, and almost no cross-peak with H7b. Taken together, these correlations can only be explained by the spatial orientation of the $6 S, 6 a S$ absolute configuration (Fig. 4c).

\section{Synergistic anti-MRSA activity of 7,8-dideoxygriseorhodin C and oxacillin}

The MICs of 1 against ESKAPE pathogens and other Grampositive bacteria were evaluated using the microdilution broth assay. Compound $\mathbf{1}$ is active in all Gram-positive bacteria tested (S. aureus, MRSA, Bacillus subtilis, Enterococcus faecium, Staphylococcus epidermidis), but not in Gram-negative bacteria (Table S2). ATCC ${ }^{\circledast} 43300^{\mathrm{TM}}$ is an oxacillin- and methicillin-resistant strain that contains staphylococcal chromosome cassette mec (SCCmec) type II encoding the mec methicillin-resistance operon. 1 has in vitro efficacy when administered as a single agent with an MIC of $0.08-0.12 \mu \mathrm{g} \mathrm{ml}^{-1}(0.12-0.25 \mu \mathrm{M})$, which was much lower than oxacillin (1.59-6.24 $\mu \mathrm{g} \mathrm{ml}^{-1}$ or 3.96-15.6 $\mu \mathrm{M})$ (Table 2). Moreover, 1 showed virtually no cytotoxicity in MDCK and AA8 (Chinese hamster ovarian cells), with $\mathrm{IC}_{50}$ of $15.84 \mu \mathrm{g} \mathrm{ml}^{-1}(32 \mu \mathrm{M})$ and $>49.5 \mu \mathrm{g} \mathrm{ml}^{-1}(100$ $\mu \mathrm{M}$ ), respectively (Fig. S7).

One strategy to decrease toxicity and slow down the development of drug resistance is through combination therapy [13]. To investigate for possible synergistic activity, ATCC $^{\circledast 4} 43300^{\mathrm{TM}}$ MRSA strain was treated simultaneously with 1 and oxacillin at $1 \times$ MIC ratio. Table 2 shows the reduction of the individual MICs after combination treatment, indicating an enhanced antimicrobial activity compared with the single-drug treatments. To assess the synergistic activity, we used the CI method developed by Chou and Talalay [18]. For antimicrobial combinations, $\mathrm{CI} \leq 0.5,>0.5-4,>4$ indicate synergism, additivity, and antagonism, respectively. The $\mathrm{CI}$ for the combination of $\mathbf{1}$ and oxacillin at MIC ratio is $0.12-0.24$, which indicates synergistic effects. This is consistent with the time-kill assay, which shows $\geq 100$-fold decrease in $\mathrm{CFU} / \mathrm{mL}$ between the combination and its most active constituent after $24 \mathrm{~h}$ (Fig. 5).

Our results show the potential renewed utility of an existing $\beta$-lactam antibiotic (oxacillin) in treatment of MRSA when used in synergy with $\mathbf{1}$. Other existing $\beta$ lactam antibiotics that are already approved for human use are also currently being restored as treatment against MRSA. Three distinct generations of $\beta$-lactam antibiotics (meropenem, piperacillin, and tazobactam) were reported to be highly synergistic against 72 clinical strains of MRSA when used as a triple combination, with FICI (Fractional Inhibitory Concentration Index) of 0.11 , while the double combinations of these drugs showed FICI of 0.22 (meropenem and piperacillin) and 0.44 (meropenem and tazobactam) against MRSA N315 (also with type II SCCmec) [25]. 


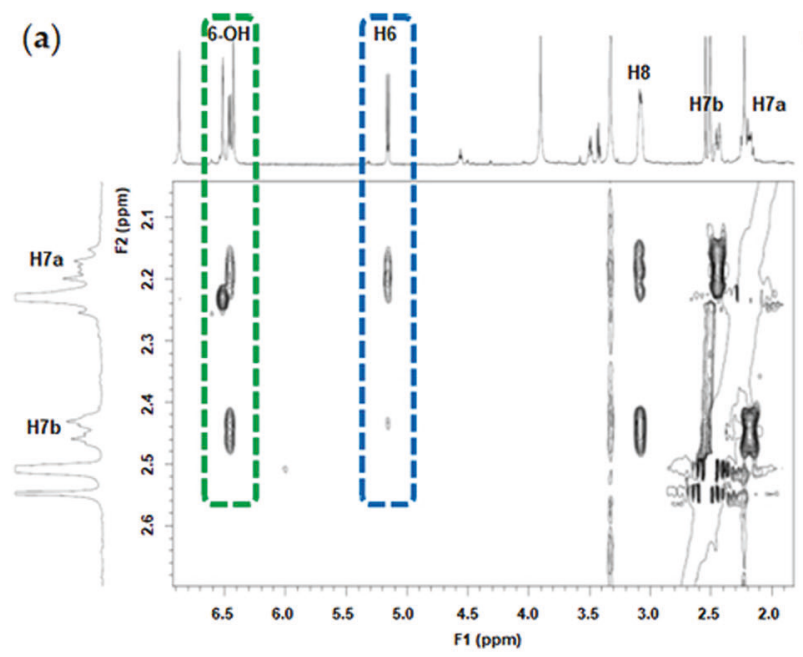

(b)

(c)<smiles>COC1=CC(=O)c2c(O)ccc(O)c2C1=O</smiles>

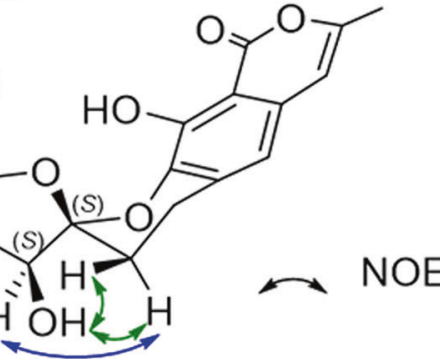

Fig. 4 ROESY correlations for determination of absolute stereochemistry. a Spectrum centered on the relevant protons. Both $\mathrm{H} 7 \mathrm{a}$ and H7b have clear ROESY cross-peaks with the hydroxyl proton 6-OH (green). These signals were in phase with cross-peaks between protons on $\mathrm{C} 7$ and $\mathrm{C} 8$, and out of phase with signals arising from exchangeable phenolic protons, indicating real ROESY correlations. Only one,

Table 2 Individual and combined effects of 7,8-dideoxygriseorhodin C (1) and oxacillin

\begin{tabular}{lcl}
\hline Individual drugs & $\begin{array}{l}\text { MIC against MRSA } \\
\left(\mu \mathrm{g} \mathrm{ml}^{-1}\right)\end{array}$ & $\begin{array}{l}\text { Combination index at } \\
1 \times \mathrm{MIC}\end{array}$ \\
\hline $\mathbf{1}$ & $0.08-0.12$ & \\
Oxacillin & $1.59-6.24$ & \\
Combination at $1 \times$ MIC ratio & $0.12-0.24$ \\
$\mathbf{1}$ & $0.01-0.02$ & \\
Oxacillin & $0.02-0.298$ & \\
\hline
\end{tabular}

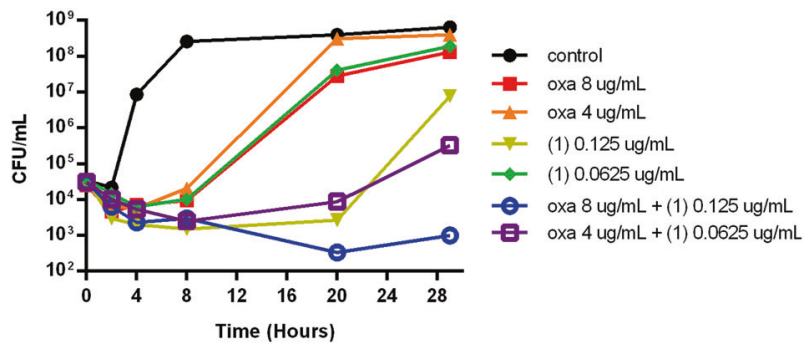

Fig. 5 Synergistic anti-MRSA activity of 7,8-dideoxygriseorhodin C and oxacillin using the time-kill assay. MRSA was treated singly or in combination with subinhibitory concentrations of 7,8-dideoxygriseorhodin $\mathrm{C}(\mathbf{1})$ and oxacillin (oxa) and $\mathrm{CFU} / \mathrm{mL}$ were determined at $0,2,4,8,20$, and $30 \mathrm{~h}$ however, shows a correlation to H6. b 3D spatial orientation of H6, 6$\mathrm{OH}, \mathrm{H} 7 \mathrm{a}$, and $\mathrm{H} 7 \mathrm{~b}$ from Gaussian generated model for $6 \mathrm{~S}, 6 \mathrm{aS}$ stereoconfiguration. In this orientation, the $6-\mathrm{OH}$ is positioned close to and approximately equidistant from the two methylene protons on carbon 7, while $\mathrm{H6}$ is oriented further from both, especially H7b. c Absolute configuration of 1 with supporting NOE correlations

ND-421, an oxadiazole, was also found to work synergistically with oxacillin in vitro with FICI values of 0.31 and 0.37 against NRS70 and NRS123 MRSA strains, respectively, as well as in vivo in a mouse neutropenic thigh model [26]. The synergistic effect is proposed to be due to simultaneous perturbation of several components of cell wall synthesis in MRSA [25, 26]. In the oxadiazole- $\beta$-lactam combination, ND-421 inhibits PBP2a [27] while oxacillin inhibits PBP2 of MRSA [26]. The transpeptidase domain of PBP2a and transglycosylase domain of PBP2 are both required in cell wall biosynthesis of MRSA in the presence of $\beta$-lactam antibiotics [28].

In conclusion, the ongoing screening of molluskassociated microbes led to the isolation of several known members of the griseorhodin structural class. One of these, 7,8-dideoxygriseorhodin C (1), demonstrated Gram-positive selective antimicrobial activity in the absence of mammalian cell toxicity. Excitingly, $\mathbf{1}$ also shows synergistic activity with the $\beta$-lactam antibiotic oxacillin, as demonstrated by the CI of $0.12-0.24$. The absolute stereochemistry of $\mathbf{1}$, which has never been previously reported, was found to be $6 S, 6 \mathrm{a} S$. It is noteworthy that the stereoconfigurations of griseorhodins $\mathrm{A}$ and $\mathrm{C}$ have been solved, and in both cases the spiroketal carbon is also in the $S$ configuration. 
However, carbon 6 was found to have the $S$ configuration in griseorhodin $\mathrm{A}$, and the $R$ configuration in griseorhodin $\mathrm{C}$. Finally, numerous inaccuracies in the literature regarding chemical shift assignments were rectified using 2D NMR experiments, as well as QM-DFT calculations.

Acknowledgements Research reported in this publication was supported by the Fogarty International Center of the National Institutes of Health under Award Number U19TW008163. The content is solely the responsibility of the authors and does not necessarily represent the official views of the National Institutes of Health. The work was completed under supervision of the Department of Agriculture-Bureau of Fisheries and Aquatic Resources, Philippines (DA-BFAR) in compliance with all required legal instruments and regulatory issuances covering the conduct of the research. We thank the Bureau of Fisheries and Aquatic Resources of the Philippines and the community of Mactan, Cebu for permission to conduct the study. We thank Dr Alexander Fedosov of A. N. Severtzov Institute of Ecology and Evolution of the Russian Academy of Science for sharing his expertise in molluscan anatomy. We also thank Jose Miguel Robes and Ryoichi Taguichi for performing some of the microbial culture, chemical extraction, and purification.

Author contributions BWM, JPT, JOT, MSF, GR, MGH, EWS, and GPC conceived and designed the experiments; BWM, JPT, JOT, MSF, and IF performed the experiments; BWM, JPT, JOT, and MSF analyzed the data; BWM, JPT, JOT, MSF, EWS, and GPC wrote the paper.

\section{Compliance with ethical standards}

Conflict of interest The authors declare that they have no conflict of interest.

Publisher's note Springer Nature remains neutral with regard to jurisdictional claims in published maps and institutional affiliations.

Open Access This article is licensed under a Creative Commons Attribution 4.0 International License, which permits use, sharing, adaptation, distribution and reproduction in any medium or format, as long as you give appropriate credit to the original author(s) and the source, provide a link to the Creative Commons license, and indicate if changes were made. The images or other third party material in this article are included in the article's Creative Commons license, unless indicated otherwise in a credit line to the material. If material is not included in the article's Creative Commons license and your intended use is not permitted by statutory regulation or exceeds the permitted use, you will need to obtain permission directly from the copyright holder. To view a copy of this license, visit http://creativecommons. org/licenses/by/4.0/.

\section{References}

1. Lin Z, Zachariah MM, Marett L, Hughen RW, Teichert RW, Concepcion GP, et al. Griseorhodins D-F, neuroactive intermediates and end products of post-PKS tailoring modification in griseorhodin biosynthesis. J Nat Prod. 2014;77:1224-30.

2. Lin Z, Torres JP, Ammon MA, Marett L, Teichert RW, Reilly CA, et al. A bacterial source for mollusk pyrone polyketides. Chem Biol 2013;20:73-81.

3. Lin Z, Flores M, Forteza I, Henriksen NM, Concepcion GP, Rosenberg G, et al. Totopotensamides, polyketide-cyclic peptide hybrids from a mollusk-associated Bacterium Streptomyces sp. J Nat Prod. 2012;75:644-9.
4. Lin Z, Reilly CA, Antemano R, Hughen RW, Marett L, Concepcion GP, et al. Nobilamides A-H, long-acting transient receptor potential vanilloid-1 (TRPV1) antagonists from molluskassociated bacteria. J Med Chem. 2011;54:3746-55.

5. Eckardt K, Tresselt D, Ihn W. The structure of the antibiotic griseorhodin C. J Antibiot. 1978;31:970-3.

6. Yang J, Fan S, Pei H, Zhu B, Xu W, Naganawa H, et al. 8-Methoxygriseorhodin $\mathrm{C}$, A new member of griseorhodin antibiotic. J Antibiot. 1991;44:1277-9.

7. Stroshane RM, Chan JA, Rubalcaba EA, Garretson AL, Aszalos AA, Roller PP. Isolation and structure elucidation of a novel griseorhodin. J Antibiot. 1979;32:197-204.

8. Suetsuna K, Seino A, Kudo T, Osajima Y. Production, and biological characterization, of dideoxygriseorhodin $\mathrm{C}$ by a Streptomyces sp. and its taxonomy. Agric Biol Chem. 1989;53:581-3.

9. Sperry J, Wilson ZE, Rathwell DCK, Brimble MA. Isolation, biological activity and synthesis of benzannulated spiroketal natural products. Nat Prod Rep. 2010;27:1117-37.

10. Stryjewski ME, Corey GR. Methicillin-resistant Staphylococcus aureus: an evolving pathogen. Clin Infect Dis. 2014;58:S10-9.

11. Tong SYC, Davis JS, Eichenberger E, Holland TL, Fowler VG. Staphylococcus aureus infections: epidemiology, pathophysiology, clinical manifestations, and management. Clin Microbiol Rev. 2015;28:603-61.

12. Worthington RJ, Melander C. Combination approaches to combat multidrug-resistant bacteria. Trends Biotechnol. 2013;31:177-84.

13. Leekha S, Terrell CL, Edson RS. General principles of antimicrobial therapy. Mayo Clin Proc. 2011;86:156-67.

14. Bruhn T, Schaumloffel A, Hemberger Y, Bringmann G. SpecDis: Quantifying the comparison of calculated and experimental electronic circular dichroism spectra. Chirality. 2013;25:243-9.

15. Weinstein MP. Methods for dilution antimicrobial susceptibility tests for bacteria that grow aerobically. 11th ed. Wayne, PA: Clinical And Laboratory Standards Institute; 2018.

16. Chou T-C, Martin N. CompuSyn. Paramus, New Jersey: ComboSyn, Inc; 2005.

17. Chou T-C. Theoretical basis, experimental design, and computerized simulation of synergism and antagonism in drug combination studies. Pharm Rev. 2006;58:621-81.

18. Chou T-C, Talalay P. Quantitative analysis of dose-effect relationships: the combined effects of multiple drugs or enzyme inhibitors. Adv Enzym Regul. 1984;22:27-55.

19. Berenbaum MC. A method for testing for synergy with any number of agents. J Infect Dis. 1978;137:122-30.

20. Mosmann T. Rapid colorimetric assay for cellular growth and survival: application to proliferation and cytotoxicity assays. J Immunol Methods. 1983;65:55-63.

21. Suetsuna K, Osajima Y. Isolation of structure of dideoxygriseorhodin C produced by a Streptomyces sp. Agric Biol Chem. 1989;53:241-2.

22. Harunari E, Imada C, Igarashi Y, Fukuda T, Terahara T, Kobayashi T. Hyaluromycin, a new hyaluronidase inhibitor of polyketide origin from marine Streptomyces sp. Mar Drugs. 2014;12:491-507.

23. Ortega HE, Batista JM, Melo WGP, Clardy J, Pupo MT. Absolute configurations of griseorhodins $\mathrm{A}$ and $\mathrm{C}$. Tetrahedron Lett. 2017;58:4721-3.

24. Yunt Z, Reinhardt K, Li A, Engeser M, Dahse H-M, Gütschow M, et al. Cleavage of four carbon-carbon bonds during biosynthesis of the griseorhodin a spiroketal pharmacophore. J Am Chem Soc. 2009;131:2297-305.

25. Gonzales PR, Pesesky MW, Bouley R, Ballard A, Biddy BA, Suckow MA, et al. Synergistic, collaterally sensitive $\beta$-lactam combinations suppress resistance in MRSA. Nat Chem Biol. 2015;11:855-61. 
26. Janardhanan J, Meisel JE, Ding D, Schroeder VA, Wolter WR, Mobashery S, et al. In vitro and in vivo synergy of the oxadiazole class of antibacterials with $\beta$-Lactams. Antimicrob Agents Chemother. 2016;60:5581-8.

27. O'Daniel PI, Peng Z, Pi H, Testero SA, Ding D, Spink E, et al. Discovery of a new class of non- $\beta$-lactam inhibitors of penicillin- binding proteins with gram-positive antibacterial activity. J Am Chem Soc. 2014;136:3664-72.

28. Łęski TA, Tomasz A. Role of penicillin-binding protein 2 (PBP2) in the antibiotic susceptibility and cell wall cross-linking of staphylococcus aureus: evidence for the cooperative functioning of PBP2, PBP4, and PBP2A. J Bacteriol. 2005;187:1815-24. 